\title{
Estrutura populacional de Uca maracoani (Decapoda, Brachyura, Ocypodidae) no Baixio Mirim, Baía de Guaratuba, Paraná
}

\author{
Mariângela Di Benedetto ${ }^{2} \&$ Setuko Masunari²
}

1. Contribuição número 1673 do Departamento de Zoologia, Setor de Ciências Biológicas da Universidade Federal do Paraná.

2. Programa de Pós-graduação em Zoologia, Departamento de Zoologia, Setor de Ciências Biológicas, Universidade Federal do Paraná, Caixa Postal 19020, 81531-980 Curitiba, PR, Brasil. (angelabe@uol.com.br; setmas@ufpr.br)

\begin{abstract}
Population structure of Uca maracoani (Decapoda, Brachyura, Ocypodidae) from the tidal flat of Baixio Mirim, Guaratuba Bay, Parana State, Brazil. A study about the annual fluctuation of the abundance, size composition, sexual proportion, reproductive period and juvenile recruitment of the fiddler crab Uca maracoani (Latreille, 1802-1803) was carried out in a population living in a tidal flat at Guaratuba Bay, Parana State, Brazil (483' W e 2552'S). Crabs were obtained from February 2005 to January 2006, during low spring tides, in monthly collections, and their carapace width was measured. Air temperature oscillated from 17 to $29^{\circ} \mathrm{C}$, luminosity from 8,740 to 151,300 lux, salinity from 8 to 25 and soil temperature (on surface, $5 \mathrm{~cm}, 10 \mathrm{~cm}, 15 \mathrm{~cm}$ and $20 \mathrm{~cm}$ depth) from 18.3 to $28.9^{\circ} \mathrm{C}$. A total of 7,120 individuals were analyzed, among them, 2,578 were sexually indefinite juveniles, 2,377 males (1,113 juveniles and 1,264 mature males) and 2,165 females (944 juveniles, 1,135 mature and 86 ovigerous females). Population abundance oscillated 341 (April) to 994 individuals (January), but its annual fluctuation was not correlated to the oscillation of the abiotic variables. The sexual proportion was 1:1 and the reproduction of the species is continuous type, with two peaks of intensity in the year: one in April and another in November. Juvenile recruitment is also continuous with two periods of intensity in the year: in July and in December-January. Sexually indefinite juveniles measured from 1.14 to $2.62 \mathrm{~mm}$ carapace width, juvenile males from 2.58 to $17.83 \mathrm{~mm}$, juvenile females from 2.60 to $11.72 \mathrm{~mm}$, mature males from 17.85 to $35.81 \mathrm{~mm}$ and mature females from 11.75 to $31.76 \mathrm{~mm}$. Males reach larger sizes than females.
\end{abstract}

KEYWORDS. Annual fluctuation, abundance, reproductive period, juvenile recruitment.

RESUMO. Um estudo sobre a flutuação anual da abundância, composição de tamanho dos indivíduos, proporção de sexos, período reprodutivo e de recrutamento dos juvenis de uma população de Uca maracoani (Latreille, 1802-1803) foi realizado no Baixio Mirim, Baía de Guaratuba, Paraná (48³6'W e 2552'S). Os animais foram coletados mensalmente, de fevereiro/2005 a janeiro/2006, durante as marés baixas de sizígia, e a sua largura da carapaça (LC) medida. A temperatura pontual do ar variou de 17 a $29^{\circ} \mathrm{C}$, a luminosidade de 8.740 a 151.300 lux, a salinidade de 8 a 25 e a temperatura do solo (superfície, $5 \mathrm{~cm}, 10 \mathrm{~cm}, 15 \mathrm{~cm}$ e $20 \mathrm{~cm}$ de profundidade) de 18,3 a $28,9^{\circ} \mathrm{C}$. Foram analisados 7.120 indivíduos, dos quais, 2.578 juvenis sexualmente indefinidos, 2.377 machos e 2.165 fêmeas. A abundância da população variou de 341 (abril) a 994 indivíduos (janeiro), mas a sua flutuação anual não esteve relacionada com a das variáveis abióticas estudadas. A proporção de sexos foi de 1:1 e a reprodução da espécie é do tipo contínuo, com dois picos de intensidade: um em abril e outro em novembro. O recrutamento de juvenis, também, é contínuo com dois períodos mais intensos no ano, um em julho e outro em dezembro-janeiro. A LC da população variou de 1,14 a 2,62 $\mathrm{mm}$ para juvenis sexualmente indefinidos, 2,58 a 17,83 mm para machos juvenis, 2,60 a 11,72 mm para fêmeas juvenis, 17,85 a 35,81 mm para machos maduros e 11,75 a 31,76 mm para fêmeas maduras. Os machos atingem tamanhos maiores do que as fêmeas.

PALAVRAS-CHAVE. Flutuação anual, abundância, período reprodutivo, recrutamento de juvenis.

Os caranguejos do gênero Uca Leach, 1814 constituem um dos grupos de Brachyura mais abundantes e mais diversos em regiões tropicais e subtropicais (Hartnoll et al., 2002). O gênero é constituído por aproximadamente 100 espécies (Rosenberg, 2001).

As espécies de $U c a$ têm como característica principal o tamanho acentuado de um dos quelípodos nos machos, levando os nomes populares de chamamaré, caranguejo violinista ou xié (COLPO \& NEGREIROSFransozo, 2004). O quelípodo aumentado não está presente nos estágios juvenis iniciais dos machos, porém o dimorfismo deste caráter já se torna aparente em juvenis ainda sexualmente imaturos, desenvolvendo-se plenamente com a maturação sexual (YAmAGUChI, 1977). Esse quelípodo não é utilizado na alimentação, mas em interações agonísticas entre os machos, na defesa de território e durante a corte
(Crane, 1975; Christy \& Salmon, 1984; Faria, 1991; Rosenberg, 1997; Backwell et al., 1999; Mariappan et al., 2000; POPE, 2000).

Os chama-marés são típicos de zonas entremarés, nas quais cavam numerosas tocas, que servem como refúgio quando os fatores físicos atingem os extremos (Powers \& Cole, 1976), refúgio de potenciais predadores e, também, de local para cópula (CRANE, 1975). São animais frequentemente simpátricos, sempre gregários, de hábito diurno e com atividade durante a maré baixa (CRANE, 1975). Suas atividades reprodutivas e comportamentais podem ser influenciadas pela interação de fatores bióticos e abióticos (temperatura, luminosidade e salinidade). Segundo Iglesia et al. (1994), que estudou U. uruguayensis Nobili, 1901, para que os indivíduos entrem em atividade plena, além da maré baixa, é necessária a presença simultânea da luz. Outro fator importante é o aumento da temperatura, 
sendo considerado como o principal fator no processo reprodutivo, por promover elevação na taxa metabólica (Negreiros-Fransozo \& Fransozo, 1992). A periodicidade reprodutiva também pode ser controlada pela combinação de fatores, incluindo latitude e zonação intertidal (EMMERSON, 1994).

O período reprodutivo é definido como o intervalo de tempo no qual as fêmeas ovígeras são encontradas na população. Certas espécies se reproduzem o ano todo, outras em uma ou mais estações do ano e ainda outras, em uma estação específica (geralmente na primavera e verão). Todos esses padrões estão relacionados com as condições ambientais locais. Brachyura da região tropical geralmente se reproduzem continuamente devido à estabilidade das variáveis abióticas (EMmERson, 1994; Negreiros-Fransozo et al., 2002).

Dentre os estudos populacionais de $U c a$ realizados nas últimas décadas, destacam-se os de Colby \& Fonseca (1984) com Uca pugilator Bosc, 1802 na Carolina do Norte, EUA, de Thurman (1985) com Uca subcylindrica Stimpson, 1859 no sul do Texas e o de Litulo (2005) com Uca urvillei H. Milne Edwards, 1852 no sul de Moçambique. No Brasil, Costa \& Negreiros-Fransozo (2002) estudaram populações de $U$. thayeri Rathbun, 1900 no manguezal de Ubatuba, SP, Colpo \& Negreiros-Fransozo (2004) as de $U$. vocator (Herbst, 1804) em três localidades do litoral norte paulista, e Косн et al. (2005), quatro espécies de Uca no manguezal do Estado do Pará, Bezerra \& Mattheus-Cascon (2006) com Uca leptodactyla Rathbun, 1898, Bezerra \& Mattheus-Cascon (2007) com U. thayeri, B ВNETTI et al. (2007) com U. burgersi Holthuis, 1967, BeDÊ et al. (2008) com oito espécies de Uca e Hirose \& Negreiros-Fransozo (2008a,b) com $U$. maracoani (Latreille, 1802-1803).

Com distribuição às margens de baías calmas do Atlântico Ocidental, U. maracoani é encontrada nas Antilhas, Venezuela, Guianas e no Brasil, do Maranhão até o Paraná (Melo, 1996). No Brasil, os seguintes estudos com $U$. maracoani foram realizados: КосH \& WOLFF (2002) sobre a estrutura da comunidade epibêntica, produção somática e fluxo energético no manguezal de estuário Caeté no norte do Brasil, $\mathrm{KocH}$ et al. (2005) no qual foi analisada a dinâmica populacional no Pará, Masunari et al. (2005) e MASUNARI (2006) sobre o crescimento relativo e a distribuição de abundância, respectivamente, no mesmo local de estudo do presente trabalho, BEZERRA et al. (2006) sobre distribuição espacial no Ceará, o de Hirose \& Negreiros-Fransozo (2007) sobre crescimento diferencial entre os sexos, Hirose \& Negreiros-Fransozo (2008a) sobre a biologia populacional no sudeste do Brasil e Hirose \& Negreiros-Fransozo (2008b) sobre o crescimento e desenvolvimento juvenil em condições laboratoriais.

Como a literatura não contempla aspectos populacionais de U. maracoani do Estado do Paraná, o presente trabalho constitui a primeira descrição da estrutura populacional de $U$. maracoani ocorrente no Baixio Mirim, no interior da Baía de Guaratuba, PR. Os seguintes aspectos são abordados: flutuação anual da abundância, composição de tamanho dos indivíduos, proporção de sexos, período reprodutivo e de recrutamento dos juvenis, com a finalidade de conhecer a influência das variáveis abióticas do litoral paranaense sobre esta população.

\section{MATERIAL E MÉTODOS}

O trabalho foi realizado no Baixio Mirim, situado na Baía de Guaratuba ( $48^{\circ} 36^{\prime} \mathrm{W}$ e 2552'S), no município do mesmo nome, Estado do Paraná. O baixio tem uma área de aproximadamente $6.300 \mathrm{~m}^{2}$ e está isolado do continente por um estreito canal de 15-20 m de largura, sendo o acesso a ele feito por meio de embarcação. Essa área tem grande influência antrópica por estar próxima à zona urbana e, frequentemente, recebe sedimento provindo dos trabalhos de dragagem do referido canal. O baixio é formado por porções de substrato arenoso não vegetado, onde predomina uma população de $U$. leptodactyla, de substrato arenoso vegetado com Spartina (Poaceae), e de substrato argiloso, no qual vive a população de $U$. maracoani. Os caranguejos foram coletados na referida área argilosa de aproximadamente $400 \mathrm{~m}^{2}$. Aves marinhas de espécies variadas pousam neste baixio e constituem os principais predadores terrestres dos mesmos.

Foram realizadas 12 amostragens mensais, de fevereiro de 2005 a janeiro de 2006, durante as marés vazantes de sizígia. Os animais foram coletados manualmente das tocas, através do revolvimento do sedimento. A abundância foi estimada em CPUE (captura por unidade de esforço) que consistiu no esforço de coleta de quatro pessoas, por 60 minutos. Estes coletores foram sempre os mesmos durante o período de estudo, tendo um deles inspecionado tocas com diâmetro de abertura de $10 \mathrm{~mm}$ ou mais, um segundo coletor, somente a superfície do lodo onde se encontravam os primeiros estágios de juvenis e dois outros coletores foram encarregados das tocas com diâmetro menores que 10 $\mathrm{mm}$.

Os caranguejos de maior porte (maiores ou iguais a $8,0 \mathrm{~mm}$ de largura cefalotorácica) foram identificados, mensurados e o sexo reconhecido ainda no campo e devolvidos ao local de captura. Foram obtidos o comprimento (CC) e largura (LC) cefalotorácicos destes caranguejos, com o auxílio de paquímetros digitais (precisão de $0,01 \mathrm{~mm}$ ). O reconhecimento do sexo foi baseado na presença de quatro pares de pleópodos no abdômen das fêmeas e um par de gonopódios nos machos, além de uma visível heteroquelia nos mesmos. Por outro lado, os juvenis pequenos (menores do que 8,0 $\mathrm{mm} \mathrm{LC}$ ) foram acondicionados em frascos de plástico, resfriados e transportados até o laboratório para posterior análise e mensuração sob microscópio estereoscópico, devido à impossibilidade de identificar a espécie e o sexo sem este instrumental. 
Medidas pontuais foram obtidas das seguintes variáveis abióticas em campo: temperatura do ar com um termômetro comum de mercúrio, luminosidade com um luxímetro, salinidade da água com um refratômetro manual e temperatura do solo à superfície, a $5 \mathrm{~cm}, 10 \mathrm{~cm}, 15 \mathrm{~cm}$ e $20 \mathrm{~cm}$ de profundidade, com um termômetro digital de campo. Estas medidas foram realizadas sempre no período da manhã, das sete e meia às oito horas, após o qual, a coleta e a mensuração dos caranguejos foram executadas. As condições climáticas por ocasião da coleta e a presença de machos de $U$. maracoani em aceno sexual também foram anotadas. As temperaturas médias mensais do ar, assim como o seu desvio padrão foram calculados a partir de temperaturas médias diárias de cada mês fornecidas pelo SIMEPAR.

Em laboratório, os juvenis dos estágios iniciais foram triados, fixados em formol $4 \%$ e conservados em álcool $75 \%$ glicerinado. Destes, os que tinham o abdômen ainda aderido ao cefalotórax foram considerados sexualmente indiferenciados. Nos estágios posteriores dos juvenis, o sexo foi reconhecido através da presença dos quatro pares de gemas de pleópodos no abdômen das fêmeas e de rudimentos do primeiro par nos machos. Estes animais foram fotografados individualmente em vista dorsal, com uma câmera fotográfica acoplada ao microscópio estereoscópico. A partir destas fotos digitais, o CC e a LC de cada juvenil foram mensurados com o auxílio do programa para computadores Jandel SigmaScan Pro 2.0.

O tamanho na primeira maturação de machos e fêmeas de 17,85 mm e de $11,75 \mathrm{~mm} \mathrm{LC}$, respectivamente, determinado por MAsunari et al. (2005) foi utilizado como referência neste estudo. Portanto, os caranguejos foram agrupados em seis categorias demográficas: juvenis sexualmente indefinidos (abdômen ainda aderido ao cefalotórax), machos juvenis (com rudimentos ou gonopódios desenvolvidos e menores ou iguais a 17,85 mm LC), machos maduros (com gonopódios desenvolvidos e maiores do que $17,85 \mathrm{~mm}$ LC), fêmeas juvenis (com quatro pares de gemas ou pleópodos desenvolvidos e menores ou iguais a 11,75 mm LC), fêmeas maduras não ovígeras e maduras ovígeras (pleópodos desenvolvidos e maiores do que $11,75 \mathrm{~mm} \mathrm{LC}$ ). Devido ao reduzido tamanho dos juvenis sexualmente indefinidos, eles foram tratados separadamente nos estudos morfométricos e foram distribuídos em cinco classes LC com 0,4 mm de amplitude cada uma, as quais foram designados pelos pontos médios 1,20 mm, 1,60 mm, 2,00 mm, 2,40 e 2,80 $\mathrm{mm}$, tanto no texto como nas figuras. Estas classes abrangeram LC de 1,01 a 3,00 $\mathrm{mm}$ e foram representados em histogramas mensais. Por outro lado, o restante dos juvenis e caranguejos maduros de ambos os sexos foram tratados trimestralmente e distribuídos em 17 classes LC com 2,0 mm de amplitude cada uma, em virtude da baixa frequência observada nas classes LC de cada mês. Cada trimestre correspondeu a uma das estações do ano: de março a maio (outono), de junho a agosto (inverno), de setembro a novembro (primavera) e de dezembro a fevereiro (verão). O número de classes LC foi estimado a partir da regra de Sturge e a determinação do intervalo das classes segundo SIEGEL (1956).

A proporção de sexos foi calculada mensalmente, sendo utilizado o teste de Qui-quadrado $\left(\chi^{2}\right)$ para a diferença de 1:1. Este teste foi utilizado, também, para avaliar a diferença de abundância mensal dos juvenis sexualmente indefinidos. No estudo da morfometria dos animais, os juvenis sexualmente indiferenciados foram analisados mensalmente, enquanto as demais categorias demográficas o foram trimestralmente. Para a análise da diferença na oscilação das temperaturas do solo nas profundidades estudadas e nos diversos meses foi utilizado o teste não paramétrico de Friedman e na comparação da oscilação das temperaturas médias mensais do ar e do solo, o teste F de Snedecor.

O período reprodutivo foi determinado pela presença de fêmeas ovígeras durante o período de amostragem e a frequência relativa de fêmeas ovígeras em relação ao total de fêmeas adultas foi calculada para cada mês.

\section{RESULTADOS}

A temperatura média mensal do ar variou de 16,75 $\pm 2,33^{\circ} \mathrm{C}$ (setembro) a $25,19 \pm 0,90^{\circ} \mathrm{C}$ (janeiro) (Fig. 1). A salinidade da água oscilou de 17 a 25 na maioria dos meses, mas houve uma redução acentuada para oito em novembro, devido à elevada pluviosidade nos dias que antecederam à coleta. A luminosidade variou de 8.740 lux (agosto) a 151.300 lux (novembro), e o tempo por ocasião da coleta apresentou-se ensolarado na maioria dos meses (em sete de um total de 12 meses); em três coletas estava nublado e em julho e outubro houve chuvisco e vento.

Nos meses mais quentes (de fevereiro a março/ 05 e de outubro/05 a janeiro/06), os valores absolutos da temperatura das camadas mais superficiais do solo foram um pouco mais altos do que os das inferiores, mas, foi constatado o inverso nos meses mais frios (maio a setembro/05) (Fig. 1). No entanto, essa diferença não foi significativa $(H=5,0279 ; \mathrm{p}>0,05)$ pelo Teste de Friedman. As temperaturas do solo (médias das profundidades) acompanharam a variação daquelas do

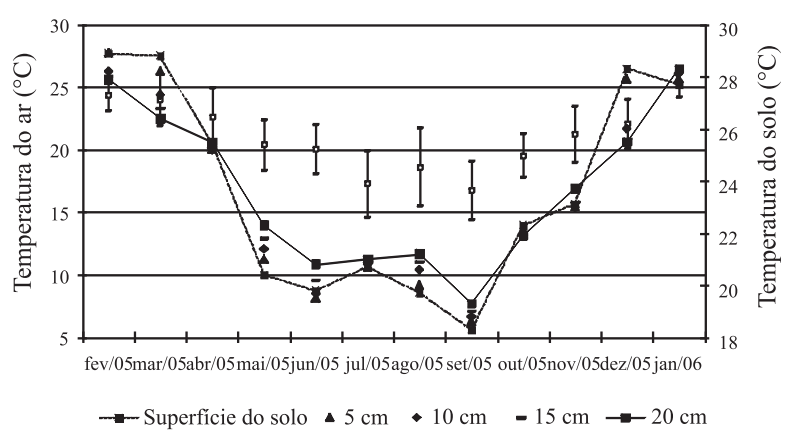

Figura 1. Flutuação anual das temperaturas médias mensais do ar (com desvio padrão) e temperaturas do solo nas diversas profundidades na Baía de Guaratuba, Estado do Paraná. 
ar (média mensal) e oscilaram de $18,8^{\circ} \mathrm{C}$ (setembro) a 28,4 ${ }^{\circ} \mathrm{C}$ (fevereiro), sem diferença significativa entre as oscilações ( $\mathrm{F}=0,4543 ; \mathrm{p}>0,05)$, pelo teste $\mathrm{F}$ de Snedecor.

Foram coletados 7.120 indivíduos de U. maracoani, dos quais 2.578 juvenis sexualmente indefinidos, 2.377 machos ( 1.113 juvenis e 1.264 maduros) e 2.165 fêmeas (944 juvenis, 1.135 maduras não ovígeras e 86 ovígeras). Todas as categorias demográficas estiveram presentes em todos os meses de amostragem (Fig. 2). Os machos realizaram aceno sexual somente nos meses mais quentes: de fevereiro a abril/05 e de setembro/05 a janeiro/06.

A flutuação anual da abundância da população mostrou pouca relação com a oscilação das variáveis abióticas analisadas (Fig. 2). Somente o pico de abundância da população $(n=994)$ ocorreu em janeiro, o mês de temperatura média do ar mais alta do ano $(25,18 \pm$ $\left.0,91{ }^{\circ} \mathrm{C}\right)$. Nos demais meses, valores baixos ou altos de abundância foram registrados com temperaturas médias altas ou baixas (Figs. 1, 2).

A abundância das várias categorias demográficas oscilou no ano sem relação com as variáveis abióticas, com exceção dos juvenis sexualmente indefinidos que mostraram relação direta com a salinidade $\left(r^{2}=0,41 ; p<\right.$ $0,05)$ e das fêmeas ovígeras com a luminosidade $\left(r^{2}=\right.$ $0,37 ; \mathrm{p}<0,05)$

Os juvenis (em conjunto os sexualmente indefinidos e juvenis de ambos os sexos) constituíram a categoria numericamente dominante em todos os meses do ano, com exceção de maio e setembro. Os primeiros variaram de 10 (nov/05) a 589 indivíduos (janeiro/06) e os últimos, de 78 (julho/05) a 390 indivíduos (outubro/05). Portanto, os juvenis sexualmente indefinidos participaram com a maior parcela dos mais de 600 juvenis registrados em janeiro (Fig. 2). Por outro lado, os indivíduos maduros variaram de 126 (abril/05) a 361 indivíduos (dezembro/ 05), mostrando que a amplitude de variação da abundância dos mesmos foi mais restrita do que a dos juvenis.

O número de machos maduros variou de 63 (fevereiro/05) a 173 indivíduos (janeiro/06), enquanto o das fêmeas maduras, de 52 (abril/05) a 199 indivíduos (dezembro/05). Por outro lado, fêmeas ovígeras ocorreram sempre em baixas frequências (sempre $<20 \%$ ) que

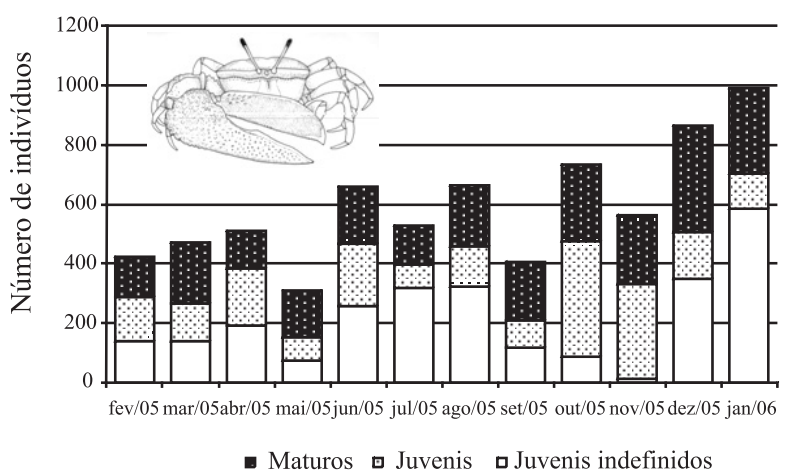

Figura 2. Distribuição dos juvenis sexualmente indefinidos, juvenis e maduros de Uca maracoani (Latreille, 1802-1803) da população no Baixio Mirim, Baía de Guaratuba, PR, durante o período de fevereiro de 2005 a janeiro de 2006 . variaram de um (maio/05) a 17 indivíduos (novembro/05), mas, mostraram dois picos de abundância no ano: o primeiro em abril/05 (19,23\%) e o segundo em novembro/ 05 (18,68\%) (Fig. 3).

A proporção de sexos (incluídos somente os juvenis sexualmente diferenciados e os maduros) foi de 1:1 (machos:fêmeas) em todos os meses, exceto em janeiro, quando ela foi de 1,4:1 mostrando uma diferença significativa com predominância de machos $\left(\chi^{2}=4,94 ; \mathrm{p}\right.$ $<0,05)$.

A LC variou de 1,14 a $2,62 \mathrm{~mm}$ para juvenis sexualmente indefinidos, de 2,58 a $17,83 \mathrm{~mm}$ para machos juvenis, de 2,60 a 11,72 mm para fêmeas juvenis, de 17,85 a $35,81 \mathrm{~mm}$ para machos maduros e de 11,75 a $31,76 \mathrm{~mm}$ para fêmeas maduras. A LC média para machos maduros $(26,49 \pm 4,37 \mathrm{~mm})$ foi superior à das fêmeas maduras $(22,28$ $\pm 4,27 \mathrm{~mm}$ ) e a das fêmeas ovígeras foi de $25,49 \pm 2,44 \mathrm{~mm}$, com amplitude de variação de 19,87 a 31,76 mm.

Os menores juvenis sexualmente indefinidos (classe $1,20 \mathrm{~mm}$ ) foram registrados em todos os meses, com exceção de setembro, outubro e novembro/ 05 , mas, houve dois picos de recrutamento: um em julho $\left(\chi_{\text {calc }}^{2}=32,8>\right.$ $\left.\chi_{(0,05 ; 11)}^{2}=19,7\right)$ e outro em dezembro/05 ( $\chi_{\text {calc }}^{2}=234,2>$ $\left.\chi_{(0,05 ; 11)}^{2}=19,7\right)$ e janeiro/06 $\left(\chi_{\text {calc }}^{2}=75,8>\chi_{(0,05 ; 1)}^{2}=19,7,\right)$ (Fig. 4). As demais categorias demográficas não mostraram um padrão claro de flutuação anual.

A distribuição de frequência de machos e fêmeas (juvenis e maduros) mostrou forte dominância de indivíduos juvenis da classe $3,5 \mathrm{~mm}$ que atingiram valores algumas vezes mais altos do que a classe modal dos adultos, especialmente na primavera (Fig. 5). A parcela juvenil mostrou histogramas assimétricos, ao passo que, os adultos, com tendência à simetria. Estes se distribuíram em histogramas unimodais no outono, inverno e primavera e bimodal no verão. Os machos atingiram tamanhos maiores do que as fêmeas e, de uma forma geral, a estrutura de tamanhos foi altamente homogênea em todas as estações do ano (Fig. 5).

As fêmeas ovígeras registradas no presente estudo foram distribuídas em sete classes LC, das quais a $23,50 \mathrm{~mm}$ foi a mais frequente, seguida da 25,50 mm e $27,50 \mathrm{~mm}$.

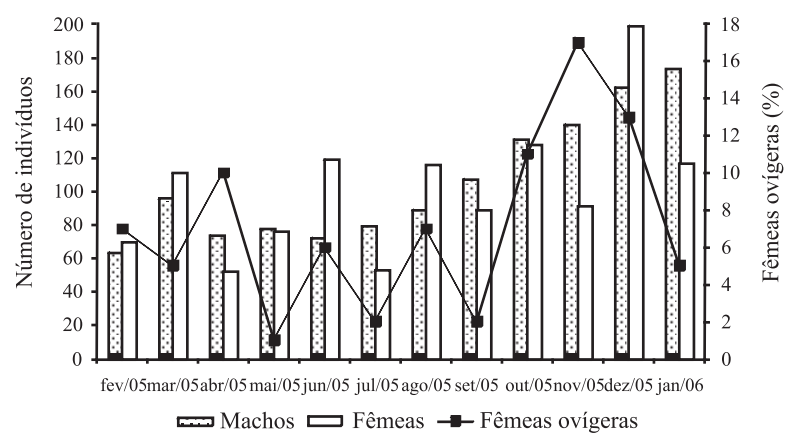

Figura 3. Frequência absoluta de machos e fêmeas e frequência relativa de fêmeas ovígeras de Uca maracoani (Latreille, 1802 1803) durante o período de fevereiro de 2005 a janeiro de 2006 , no Baixio Mirim, Baía de Guaratuba, PR. 
Fev/05

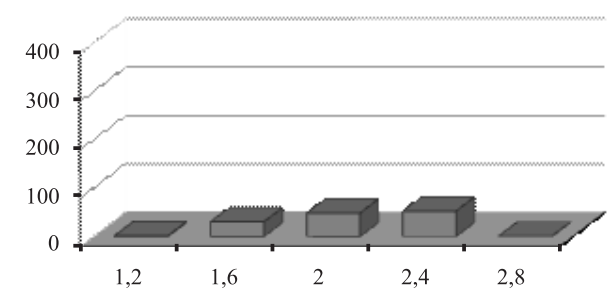

$\mathrm{Mar} / 05$

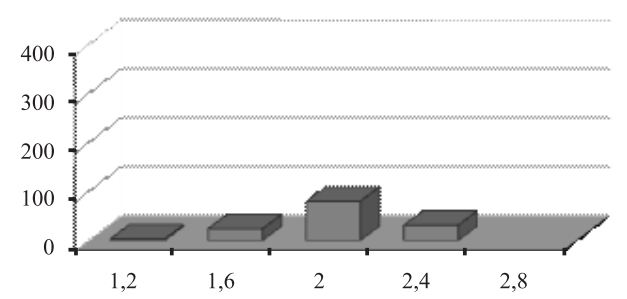

$\mathrm{Abr} / 05$

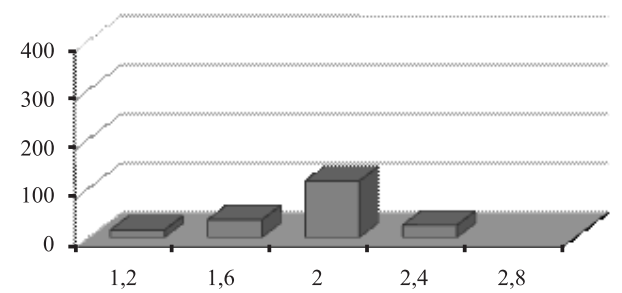

Mai/05

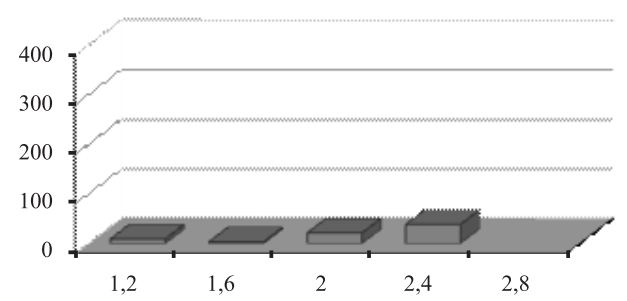

Jun/05

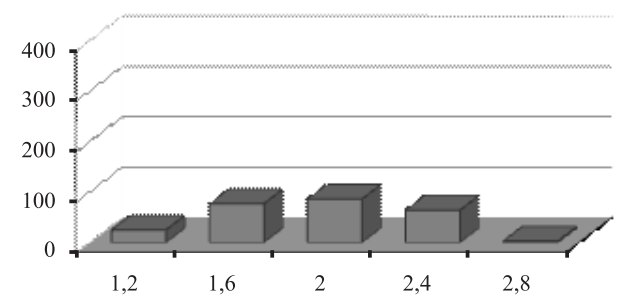

$\mathrm{Jul} / 05$

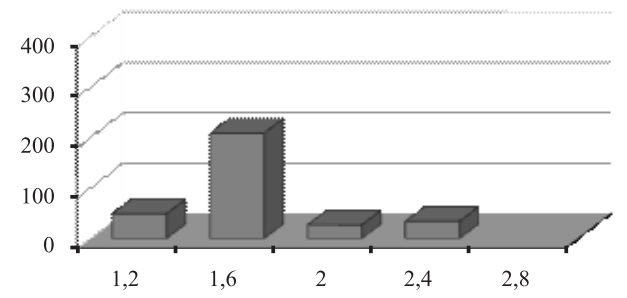

Ago/05

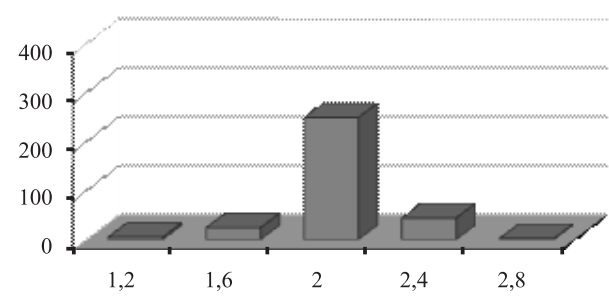

Set/05

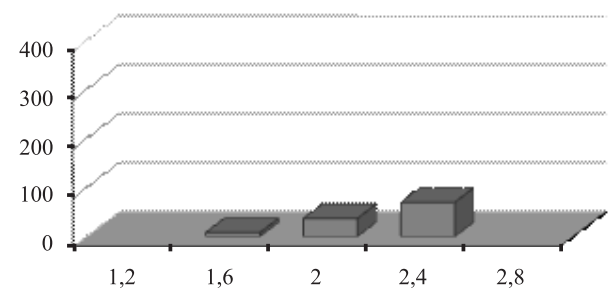

Out/05

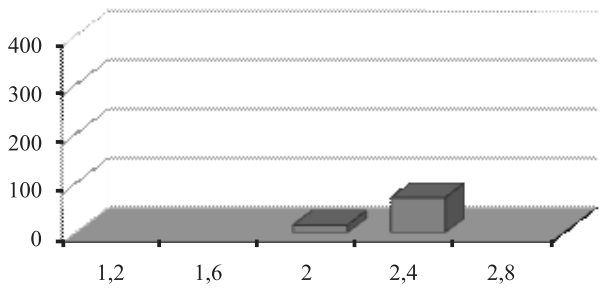

Nov/05

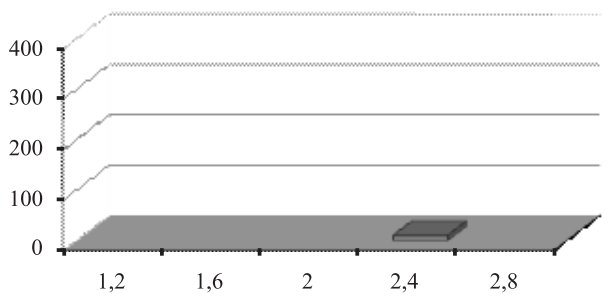

Dez/05

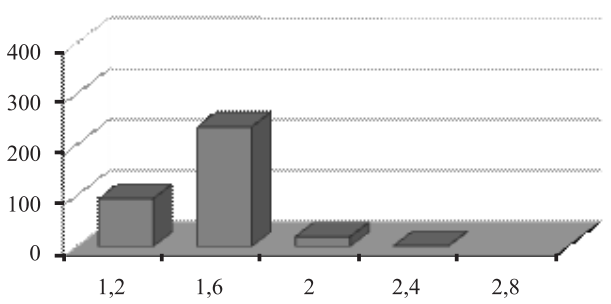

$\operatorname{Jan} / 06$

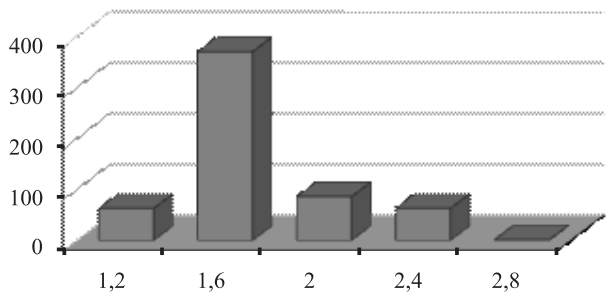

Figura 4. Distribuição de frequência absoluta de juvenis sexualmente indefinidos de Uca maracoani (Latreille, 1802-1803) nas classes de largura da carapaça, durante o período de fevereiro de 2005 a janeiro de 2006, no Baixio Mirim, Baía de Guaratuba, PR. 


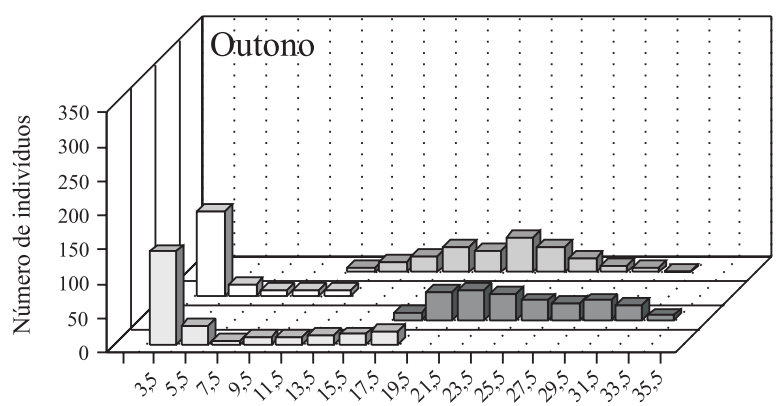

Classes de largura da carapaça $(\mathrm{mm})$

$\square$ Machos Juvenis $\square$ Machos Maduros $\square$ Fêmeas Juvenis $\square$ Fêmeas Maduras

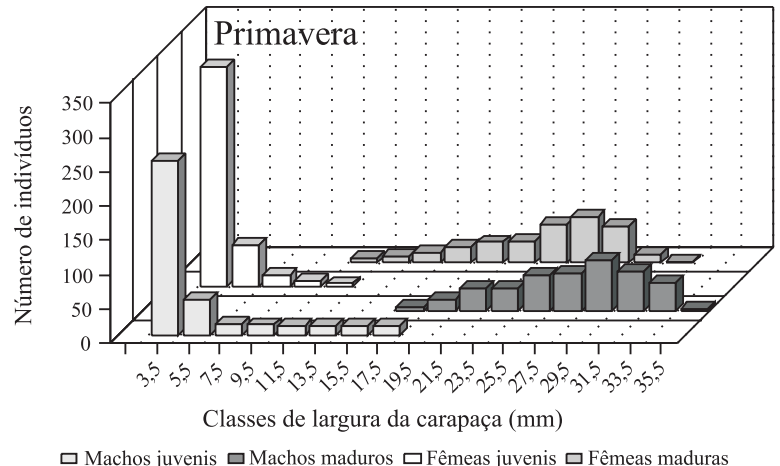

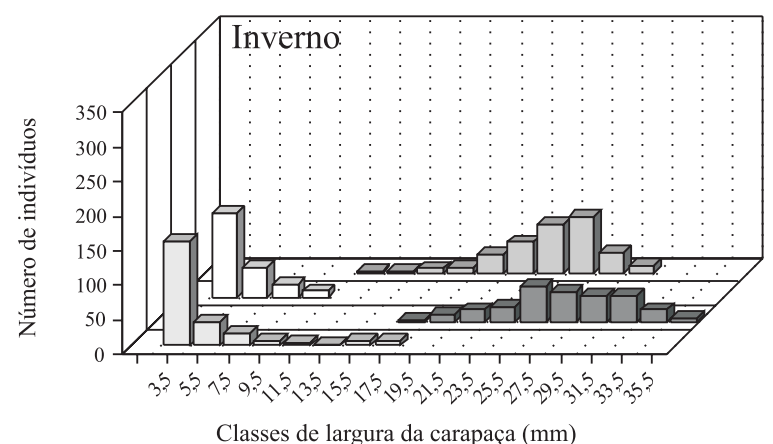

$\square$ Machos juvenis $\square$ Machos maduros $\square$ Fêmeas juvenis $\square$ Fêmeas maduras

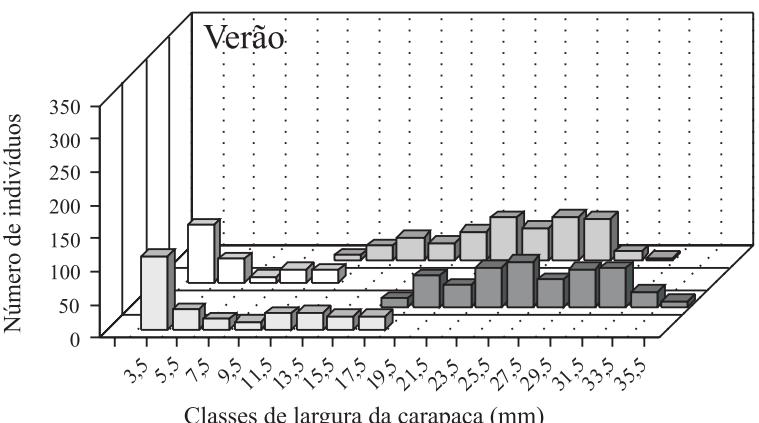

$\square$ Machos juvenis $\square$ Machos maduros $\square$ Fêmeas juvenis $\square$ Fêmeas maduras

Figura 5. Distribuição de frequência absoluta de juvenis e adultos de ambos os sexos de Uca maracoani (Latreille, 1802-1803) nas classes de largura da carapaça, nas quatro estações do ano, no Baixio Mirim, Baía de Guaratuba, PR.

\section{DISCUSSÃO}

No presente estudo, a falta de correlação entre a flutuação anual da abundância de $U$. maracoani e a da salinidade e temperatura do ar ressalta a natureza eurihalina (amplitude de oscilação da salinidade de 8 a 25) e euritérmica (amplitude de oscilação da temperatura do ar de cerca de $15^{\circ} \mathrm{C}$ ) da espécie. Tampouco, a ampla oscilação da intensidade luminosa e a variação de cerca de $10^{\circ} \mathrm{C}$ na temperatura do solo influíram no referido parâmetro populacional. A relação direta entre a flutuação da salinidade com a abundância dos juvenis sexualmente indefinidos pode ser explicada pela influência das marés que deve ter sido de maior intensidade sobre esta categoria demográfica, já que a maioria deles encontravase ainda na superfície do solo, certamente à procura de um local adequado para a construção de suas tocas. Por outro lado, a relação direta entre a flutuação da luminosidade e a das fêmeas ovígeras é de difícil interpretação, pois elas nunca foram vistas fora das tocas e, portanto, elas não estavam expostas à ação direta dos raios solares.

Os valores médios de temperatura do solo mais altos do que os do ar registrados no presente estudo, especialmente em dias ensolarados do verão, podem ser explicados pelo horário de medida deste parâmetro: sempre durante o dia, com incidência direta dos raios solares na superfície do solo. Entretanto, como as temperaturas do solo oscilaram no mesmo padrão com as médias do ar, é muito provável que $U$. maracoani não procure as tocas no solo como refúgio contra as oscilações de temperatura do ar. Em outras populações da espécie, as adversidades de temperaturas extremas são enfrentadas pela permanência dos indivíduos no interior das tocas (Косн et al., 2005).

A caracterização estrutural de populações constitui informação fundamental para medidas que visem à manutenção dos recursos naturais. Além disso, tais informações podem ser empregadas em trabalhos de cunho ecológico, pois, tratam de assuntos relacionados com a natalidade, a mortalidade, o crescimento e a migração (HutCHINSON, 1981).

A proporção de sexos é muito variável entre as espécies do gênero $U c a$. Em algumas, as fêmeas são predominantes como em $U$. pugilator, Uca cumulanta Crane, 1943 e U. rapax (Smith, 1870) (Colby \& FonseCA, 1984; Косн et al., 2005). No entanto, os machos são dominantes na maioria das espécies cuja proporção de sexos é conhecida: em Uca pugnax Smith, 1870, U. uruguayensis, Uca lactea annulipes $\mathrm{H}$. Milne Edwards, 1837, U. urvillei, Uca vocans herperiae Crane, 1975, Uca chlorophthalmus chlorophthalmus H. Milne-Edwards, 1852, U. vocator e U. urvillei (Wolf et al., 1975; SPIVAK et al., 1991; Emmerson, 1994; Colpo \& NegreirosFransozo, 2004; Litulo, 2004, 2005). Além disso, esta proporção pode variar conforme a metodologia de amostragem, como foi registrada por CosTA \& NEGREIROSFRANSOZO (2002) para U. thayeri, os quais obtiveram uma proporção de 1:1 quando amostraram a população através da técnica de CPUE, e de dominância de machos, pela técnica de amostragem por transecção. Devido a estes artifícios de amostragem, SKOv \& HARTNOLL (2001) 
já haviam sugerido que o melhor método é o de escavação do substrato, pois, é o que apresenta resultados mais próximos do número real de chama-marés. Segundo os referidos autores, uma coleta dos animais presentes na superfície ou por contagem direta com auxílio de binóculos levaria a uma superestimativa de machos, em função destes serem mais ativos e mais visíveis devido ao maior tamanho do corpo e da presença do quelípodo gigante. Embora no presente estudo, os animais tenham sido coletados exclusivamente por inspeção das tocas, o alto número de exemplares examinados representou uma situação real da população do Baixio Mirim. Кoch et al. (2005) também encontraram uma proporção de sexos de 1:1 para uma população de $U$. maracoani ocorrente no Manguezal do Caeté, PA, indicando que, nesta espécie, uma eventual competição entre os machos por fêmeas durante o período de acasalamento deve ser fraca ou inexistente.

Adicionalmente, a diferença na proporção de sexos, em muitas populações, pode estar relacionada a diversos fatores como: diferentes taxas de mortalidade (GENONI, 1985), padrões de migração (MonTAGUE, 1980) e maior facilidade de um dos sexos em enfrentar adversidades ambientais, diferenciação temporal ou espacial na utilização de recursos do habitat e padrões comportamentais diferenciados entre os sexos (CHRISTY \& SALMON, 1984).

Como principais fatores bióticos e abióticos controladores do início e duração do período reprodutivo em Crustacea são citadas a latitude, a temperatura, a disponibilidade alimentar para as larvas e a zonação intertidal (Pillay \& ONO, 1978; SAStRY, 1983; Costa \& Negreiros-Fransozo, 2002). O período reprodutivo dos chama-marés tende a ser mais longo em espécies de regiões subtropicais mais quentes do que nas mais frias e, em regiões temperadas, a reprodução geralmente é sazonal (EMMERSon, 1994). Pelo fato de estarem adaptados a viver em climas quentes (trópicos e subtrópicos quentes), os chama-marés em atividade reprodutiva são encontrados durante o ano todo, se as condições ambientais forem favoráveis à alimentação, ao desenvolvimento gonadal e à liberação das larvas (SASTRY, 1983; Thurman, 1985). No entanto, existem espécies de Uca tropicais ou subtropicais que apresentam reprodução sazonal com maior intensidade reprodutiva nos meses mais quentes do ano, como observado em $U$. pugilator, $U$. uruguayensis, U. vocans herperiae, U. c. chlorophthalmus, $U$. lactea annulipes, $U$. thayeri, $U$. spinicarpa Rathbun, 1900 e Uca longisignalis Salmon \& Atsaides, 1968 (Colby \& Fonseca, 1984; SpivaK et al., 1991; Emmerson, 1994; Costa et al., 2006; Mouton \& FELDER, 1995).

No presente estudo, a ocorrência constante de fêmeas ovígeras de $U$. maracoani em todos os meses indica uma reprodução contínua no ano com recrutamento de juvenis o ano inteiro, de acordo com SASTRY (1983). Somente a intensidade reprodutiva não foi constante entre os meses, pois, houve dois picos de abundância de fêmeas ovígeras no ano e dominância de juvenis em dois períodos. Pode-se inferir que os juvenis do primeiro período de dominância (junho a agosto) correspondem às larvas eclodidas de fêmeas ovígeras ocorrentes no mês de pico de abundância (abril), ao passo que os do segundo período de dominância (dezembro a janeiro), às do pico de novembro. Este fato sugere que o desenvolvimento larval (zoeas e megalopa) ocorre num intervalo muito curto de aproximadamente dois a três meses, como foi observado por RIEGER (1996, 1997, 1998, 1999) com várias espécies de Uca.

O registro de machos em aceno sexual limitado aos meses de setembro a abril, leva a inferir que o acasalamento ocorre somente neste período. Portanto, o recrutamento contínuo de juvenis no ano é de difícil interpretação, a não ser que estudos sobre uma possível reserva de gametas masculinos no sistema reprodutivo das fêmeas sejam realizados. Estas realizariam a fertilização e a postura dos ovos para os pleópodos em qualquer época do ano, independentemente do período de aceno sexual dos machos. Esta estratégia garantiria o recrutamento contínuo de juvenis observado no presente estudo, apesar da limitação no período de acasalamento.

Outro fator que pode influenciar na sazonalidade da reprodução é o regime de chuvas, sendo que em algumas espécies de regiões subtropicais, a reprodução é influenciada pela intensidade da precipitação, mais do que a temperatura (CRANE, 1975). Tal resultado foi obtido por Koch et al. (2005) que observaram ocorrência de fêmeas ovígeras com dois picos de intensidade no período mais seco do ano (junho a dezembro) e supressão das mesmas no período chuvoso, no manguezal do Pará. Entretanto, o registro de recrutamento contínuo de juvenis neste local permite inferir que as fêmeas foram subamostradas em função do declínio na abundância das mesmas no período chuvoso. A ocorrência contínua de fêmeas ovígeras no Baixio Mirim indica que o regime de chuvas neste local não influi neste parâmetro populacional. Portanto, U. maracoani tem reprodução contínua no ano, com dois picos de intensidade, independentemente da localização geográfica.

A estrutura populacional dos crustáceos tem sido geralmente analisada pela distribuição dos indivíduos em classes de tamanho, razão de sexos, dinâmica temporal, crescimento, taxa de natalidade e mortalidade (NAKAGAKI \& PinheIro, 1999). O presente estudo revelou a presença de todas as categorias de largura da carapaça em todo o período amostrado, com exceção de alguns estágios juvenis sexualmente indefinidos, que estiveram ausentes em alguns meses. Isto corrobora a hipótese de que espécie se reproduz continuamente, embora em taxas diferenciadas no ano.

Uca maracoani é a espécie que atinge LC máximo mais elevado dentre aquelas do gênero $U c a$ ocorrentes no Brasil e, no exterior, ela é ultrapassada somente por Uca tangeri Eydoux, 1835 do litoral da África, Uca ornata Smith, 1870 do Panamá e Uca insignis H. Milne-Edwards, 1852 do Equador (Crane, 1975).

Os LC máximos de populações de $U$. maracoani variam dependendo do local habitado pela espécie. Para uma população do Equador, foram de 46,5 $\mathrm{mm}$ LC para machos e de $36,5 \mathrm{~mm}$ LC para fêmeas e para outra de Georgetown, Guiana foram de 42,5 mm LC e 33,0 mm LC, respectivamente (CRANE, 1975). Para a população estudada por Косн et al. (2005) no Estado do Pará foram de 35,2 $\mathrm{mm} L \mathrm{C}$ para machos e $31 \mathrm{~mm} \mathrm{LC}$ para fêmeas. Estes valores, 
quando comparados com os da população da Baía de Guaratuba (machos: $35,81 \mathrm{~mm} \mathrm{LC}$ e fêmeas: $31,76 \mathrm{~mm} \mathrm{LC}$ ), mostram que tanto os machos da população de Georgetown quanto os do Equador crescem mais que os do presente estudo e os do Pará, que têm valores muito próximos. As diferenças nas dimensões corporais de uma mesma espécie estão relacionadas a variados fatores, dentre os quais citam-se a latitude do local de ocorrência, qualidade do habitat e a quantidade de matéria orgânica presente no substrato (Masunari \& DisSenha, 2005).

A distribuição trimestral da população de $U$. maracoani nas classes de LC mostrou que a espécie possui um padrão relativamente estável na sua composição durante o ano todo, sobretudo os adultos que mostraram uma distribuição unimodal em ambos os sexos, na maior parte do ano. Os juvenis são dominantes em todos os trimestres, sobretudo na primavera, e as altas taxas de mortalidade entre esta categoria demográfica e os adultos caracterizam uma população de altas taxas de mortalidade durante o desenvolvimento.

Os machos que ocuparam classes de LC maiores do que as fêmeas em todos os trimestres amostrados no presente estudo indicam que elas crescem menos do que eles: observação amplamente registrada em outras espécies do gênero Uca (ColBy \& FonSECA, 1984; SPIVAK et al., 1991; Litulo, 2005; CAstiglioni \& NegreirosFRANSOZO, 2006). Este fato pode estar relacionado com a necessidade das fêmeas de dividir seus recursos energéticos entre a produção de ovócitos e o crescimento (HARTNOLL, 1982).

$\mathrm{O}$ registro de fêmeas ovígeras distribuídas mais frequentemente na classe de $\mathrm{LC}$ de $23,50 \mathrm{~mm}$ permite deduzir que a espécie atinge a fertilidade máxima bem antes de atingir o seu tamanho máximo que é a classe de LC de $31,50 \mathrm{~mm}$. As fêmeas das classes de LC maiores do que esta última (todas não ovígeras) somaram mais de 100 indivíduos e, como podem ser consideradas totalmente estéreis, representam o contingente em senescência da população.

Agradecimentos. Ao Coordenador do Programa de Pósgraduação em Zoologia da UFPR, pela permissã̃o no uso das instalações e equipamentos. Ao CNPq pela bolsa de mestrado à primeira autora e de produtividade à segunda. Ao SIMEPAR pela cessão das temperaturas médias mensais do ar. À Profa. Dra. Sônia Graça Melo, pelas sugestões e colaboração. Ao M.Sc. Érico Augusto F. M. O. Teodósio pelo auxílio nos trabalhos de campo e de laboratório.

\section{REFERÊNCIAS BIBLIOGRÁFICAS}

Backwell, P. R. Y.; Jennions, M. D.; Christy, J. H. \& Passmore, N. I. 1999. Female choice in the synchronously waving fiddler crab Uca annulipes. Ethology 105:415-421.

Bedê L. M.; Oshiro L. M. Y.; Mendes L. M. D. \& Silva A. A. 2008. Comparação da estrutura populacional das espécies de Uca (Crustacea: Decapoda: Ocypodidae) no Manguezal de Itacuruçá, Rio de Janeiro, Brasil. Revista Brasileira de Zoologia 25(4):601-607.

Benetti A. S.; Negreiros-Fransozo, M. L. \& Costa T. M. 2007. Population and reproductive biology of the crab Uca burgersi (Crustacea: Ocypodidae) in three subtropical mangrove forests. Revista de Biología Tropical 55(1): 55-70.

Bezerra, L. E. A.; Dias, C. B.; Santana, G. X. \& Matthews-Cascon, H. 2006. Spatial distribution of fiddler crabs (genus Uca) in a tropical mangrove of northeast Brazil. Scientia Marina 70(4):759-766
Bezerra, L. E. A. \& Mattheus-Cascon, H. 2006. Population structure of the fiddler crab Uca leptodactyla Rathbun, 1898 (Brachyura: Ocypodidae) in a tropical mangrove of northeast Brazil. Thalassas 22(1):65-75.

2007. Population and reproductive biology of the fiddler crab Uca thayeri Rathbun, 1900 (Crustacea: Ocypodidae) in a tropical mangrove from Northeast Brazil. Acta Oecologica 31(3):251-258.

Castiglioni, D. S. \& Negreiros-Fransozo, M. L. 2006. Ciclo reprodutivo do caranguejo violinista Uca rapax (Smith) (Crustacea, Brachyura, Ocypodidae) habitante de um estuário degradado em Paraty, Rio de Janeiro, Brasil. Revista Brasileira de Zoologia 23(2):331-339.

Christy, J. H. \& SALmon, M. 1984. Ecology and evolution of mating systems of fiddler crabs (genus Uca). Biological Review (Cambridge) 59:483-509.

Colby, D. R. \& FonseCA, M. S. 1984. Population dynamics, spatial dispersion and somatic growth of the sand fiddler crab Uca pugilator. Marine Ecology Progress Series 16:269-279.

Colpo, K. D. \& Negreiros-Fransozo, M. L. 2004. Comparison of the population structure of the fiddler crab Uca vocator (Herbst, 1804) from three subtropical mangrove forests. Scientia Marina 68(1):139-146.

Costa, T. M. \& Negreiros-Fransozo, M. L. 2002. Population biology of Uca thayeri Rathbun, 1900 (Brachyura, Ocypodidae) in a subtropical South American mangrove area: results from transect and catch-per-unit-effort techniques. Crustaceana 75(10): 1201-1218

Costa, T. M.; Silva, S. M. J. \& Negreiros-Fransozo, M. L. 2006 Reproductive pattern comparison of Uca thayeri Rathbun, 1900 and Uca uruguayensis Nobili, 1901 (Crustacea, Decapoda, Ocypodidae). Brazilian Archives of Biology and Technology 49(1):117-123.

Crane, J. 1975. Fiddler crabs of the world, Ocypodidae: genus Uca. Princeton, Princeton University. 736p.

EMmerson, W. D. 1994. Seasonal breeding cycles and sex ratio of eight species of crabs from Magazana, a mangrove estuary in Transkei, southern Africa. Journal of Crustacean Biology 14(3):568-578.

FARIA, M. M. 1991. A luta e o dimorfismo masculino nos caranguejos violinistas Uca tangeri (Eydoux), (Ocypodidae, Brachyura). Actas do I Congresso Nacional de Etologia 127-139.

Genoni, G. P. 1985. Food limitation in salt marsh fiddler crabs Uca rapax (Smith) (Decapoda: Ocypodidae). Journal of Experimental Marine Biology and Ecology 87:97-110

Hartnoll, R. G. 1982. Growth. In: Abele, L. G. ed. The biology of Crustacea, embriology, morphology and genetics. New York, Academic. v.2, p.111-196.

Hartnoll, R. G.; Cannicci, S.; Emmerson, W. D.; Fratini, S.; Macia, A.; Mgaya, Y.; Porri, F.; Ruwa, R. K.; Shunula, J. P.; Skov, M. W. \& VANninI, M. 2002. Geographic trends in mangrove crab abundance in East Africa. Wetlands Ecology and Management 10:203-213.

Hirose, G. L. \& Negreiros-Fransozo, M. L. 2007. Growth phases and diferential growth between sexes of Uca maracoani Latreille, 1802-1803 (Crustacea, Brachyura, Ocypodidae). Gulf and Caribbean Research 19:43-50.

2008a. Population biology of Uca maracoani Latreille 1802-1803 (Crustacea, Brachyura, Ocypodidae) on the southeastern coast of Brazil. Pan-American Journal of Aquatic Sciences 3:373-383.

$2008 \mathrm{~b}$. Growth and juvenile development of Uca maracoani Latreille 1802-1803 in laboratory conditions. Senckenbergiana Biologica 88:161-168.

Hutchinson, G. E. 1981. Introducción a la ecologia de poblaciones. Barcelona, Editorial Blume. 492p.

Iglesia, H. O.; Rodriguez, E. M. \& Dezi, R. E. 1994. Burrow plugging in the crab Uca uruguayensis and its synchronization with photoperiod and tides. Physiology and Behavior 55(5):913-919.

Koch, V. \& WolfF, M. 2002. Energy budget and ecological role of mangrove epibenthos in the Caeté estuary, North Brazil. Marine Ecology Progress Series 228:119-130.

Koch, V.; WolfF, M. \& Diele, K. 2005. Comparative population dynamics of four fiddler crabs (Ocypodidae, genus Uca) from 
a North Brazilian mangrove ecosystem. Marine Ecology Progress Series 291:177-188.

Litulo, C. 2004. Reproductive aspects of a tropical population of the fiddler crab Uca annulipes (H. Milne Edwards, 1837) (Brachyura: Ocypodidae) at a Costa do sol Mangrove, Maputo Bay, southern Mozambique. Hydrobiologia 525:167-173. 2005. Population structures and reproductive biology of the fiddler crab Uca urvillei (Brachyura: Ocypodidae) in Maputo Bay (south Mozambique). Journal of Natural History 39(25):2307-2318.

Mariappan, P.; Balasundaram, C. \& Schimitz, B. 2000. Decapod crustacean chelipeds: an overview. Journal of Bioscience 25(3):301-313

Masunari, S. 2006. Distribuição e abundância dos caranguejos Uca Leach (Crustacea, Decapoda, Ocypodidae) na Baía de Guaratuba, Paraná, Brasil. Revista Brasileira de Zoologia 23(4):901-914.

Masunari, S. \& Dissenha, N. 2005. Alometria no crescimento de Uca mordax (Smith) (Crustacea, Decapoda, Ocypodidae) na Baía de Guaratuba, Paraná, Brasil. Revista Brasileira de Zoologia 22(4):984-990.

Masunari, S.; Dissenha, N. \& Falcão, R. C. 2005. Crescimento relativo e destreza dos quelípodos de Uca maracoani (Latreille) (Crustacea, Decapoda, Ocypodidae) no Baixio Mirim, Baía de Guaratuba, Paraná, Brasil. Revista Brasileira de Zoologia 22(4): $974-983$.

Melo, G. A. S. 1996. Manual de identificação dos Brachyura (caranguejos e siris) do litoral brasileiro. São Paulo, Plêiade/FAPESP. 604p.

Montague, C. L. 1980. A natural history of temperate western Atlantic fiddler crabs (genus $U c a$ ) with reference of their impact on the salt marsh. Contributions in Marine Science 23:25-55.

Mouton, E. C. \& Felder, D. L. 1995. Reproduction of the fiddler crabs Uca longisignalis and Uca spinicarpa in a Gulf of Mexico Salt Marsh. Estuaries 18(3):469-481.

Nakagaki, J. M. \& Pinheiro, M. A. 1999. Biologia populacional de Emerita brasiliensis Schmitt (Crustacea, Hippidae) na Praia Vermelha do Norte, Ubatuba (São Paulo, Brazil). Revista Brasileira de Zoologia 16(2):83-90.

Negreiros-Fransozo, M. L. \& Fransozo, A. 1992. Estrutura populacional e relação com a concha em Paguristes tortugae Schimitt, 1933 (Decapoda, Diogenidae), no litoral norte do Estado de São Paulo, Brasil. Naturalia 17:31-42.

Negreiros-Fransozo, M. L.; Fransozo, A. \& Bertini, G. 2002. Reproductive cycle and recruitment period of Ocypode quadrata (Decapoda, Ocypodidae) at a sandy beach in southeastern Brazil. Journal of Crustacean Biology 22(1):157-161.

Pillay, K. K. \& Ono, Y. 1978. The breeding cycles of two species of grapsid crabs (Crustacea: Decapoda) from the north coast of Kyushu, Japan. Marine Biology 45:237-248.

Pope, D. S. 2000. Testing function of fiddler crab claw waving by manipulating social context. Behavioral Ecology Sociobiology 47:432-437.

Powers, L. W. \& Cole, J. F. 1976. Temperature variation in fiddler crab microhabitats. Journal of Experimental Marine Biology and Ecology 21:141-157.

Rieger, P. J. 1996. Desenvolvimento larval de Uca (Celuca) uruguayensis Nobili, 1901(Crustacea, Decapoda, Ocypodidae), em laboratório. Nauplius 4:73-103.

1997. Desenvolvimento larval de Uca (Minuca) mordax (Smith, 1870) (Crustacea, Decapoda, Ocypodidae), em laboratório. Trabalhos Oceanográficos da Universidade Federal de Pernambuco 25:227-267.

1998. Desenvolvimento larval de Uca (Minuca) burgersi Holthuis(Crustacea, Decapoda, Ocypodidae), em laboratório. Revista Brasileira de Zoologia 15(3):727-756.

1999. Desenvolvimento larval de Uca (Minuca) vocator (Herbst, 1804) (Crustacea, Decapoda, Ocypodidae), em laboratório. Nauplius 7:1-37.

RosenberG, M. S. 1997. Evolution of shape differences between the major and the minor chelipeds of Uca rapax (Decapoda: Ocypodidae). Journal of Crustacean Biology 17:52-59.

. 2001. The systematics and taxonomy of fiddler crabs: a phylogeny of the genus Uca. Journal of Crustacean Biology 21(3):839-869.

SASTRY, A. N. 1983. Ecological aspects of reproduction. In: Venberg, W. B. ed. The Biology of Crustacea: Environmental adaptations. New York, Academic. p.179-270.

SIEGEL, S. 1956. Estatística não paramétrica para as ciências do comportamento. Rio de Janeiro, McGraw-Hill. 350p.

Skov, M. W. \& Hartnoll, R. G. 2001. Comparative suitability of binocular observation, burrow counting and excavation for the quantification of the mangrove fiddler crab Uca annulipes (H. Milne Edwards). Hydrobiologia 449:201-212.

Spivak, E. D.; Gavio, M. A. \& Navarro, C. E. 1991. Life history and structure of the world's southernmost Uca population: Uca uruguayensis (Crustacea, Brachyura) in Mar Chiquita Lagoon (Argentina). Bulletin of Marine Science 48(3):679-688.

Thurman, C. L. 1985. Reproductive biology and population structure of the fiddler crab Uca subcylindrica (Stimpson). Biological Bulletin 169:215-229.

Wolf, P. L.; Shanholtzer, S. F. \& Reimold, R. J. 1975. Population estimates for Uca pugnax (Smith, 1870) on the Duplin Estuary Marsh, Georgia, USA (Decapoda, Brachyura, Ocypodidae). Crustaceana 29:79-91.

YamaGuChI, T. 1977. Studies on the handedness of the fiddler crab, Uca lactea. Biological Bulletin 152:424-436. 